\title{
Optimized load balancing for efficient resource provisioning in the cloud
}

\begin{abstract}
Cloud Computing offers on demand provisioning of computing resources to users. Cloud service providers manage a large number of user requests to provide services according to user demands. Allocating and managing user requests to physical hardware is a challenging issue, because there is a need to create a load balance among available system resources. Effective load balancing saves operational costs, improves user satisfaction and leads to accelerate overall performance. In this paper, we propose an algorithm entitled Optimized Load Balancing (OLB), which aims to carry out efficient load balancing by improving processing and response time. We compared our proposed load balancing algorithm with an existing load balancing algorithm. Experimental results show that our proposed algorithm outperforms the existing one.
\end{abstract}

Keyword: Cloud computing; Cloud simulation; Data center; Load balancing; Response time 